\title{
INITIATION A LA TECHNOLOGIE D'IMPRESSION JET D'ENCRE : \\ Réalisation de jauges de contraintes organiques sur papier
}

M. HARNOIS*, T. MOHAMMED BRAHIM, E. JACQUES

CCMO, Centre commun de microélectronique de l'OUEST, GIP-CNFM, Université de Rennes 1

Campus de Beaulieu, 35042 Rennes cedex, France

\section{INTRODUCTION}

Ce TP a pour but de sensibiliser des étudiants à la réalisation de dispositifs par la technologie d'impression jet d'encre. La réalisation de capteurs de déformation mécanique de type jauge de contrainte constitue un bon support pédagogique de par sa simplicité pour appréhender les notions essentielles inhérentes à cette technologie. L'impression de composants électroniques sur substrat flexible est une thématique aujourd'hui très porteuse, nous devons donc former nos étudiants à cette problématique. Le choix d'un substrat flexible autre que des plastiques (PEN, PET, Polyimide), tel que du papier semble original car encore peu étudié. D'autre part, la technologie d'impression jet d'encre encore en phase de développement constituera une technique de choix dans la réalisation de dispositifs électronique à grande échelle.

\section{REALISATION TECHNOLOGIQUE :}

La réalisation technologique s'effectuera au sein de la plateforme technologique du CCMO (Centre Commun de Microélectronique de l'Ouest) dans le groupe microélectronique et microcapteurs de l'IETR (Institut d'Electronique et des Télécommunications de Rennes). Ce dernier a acquis depuis peu un équipement d'impression jet d'encre CERADROP de type Xseries multimatériaux (figure 1) permettant d'imprimer des encres à base de nanoparticules. Dans ce TP, des nanoparticules d'argent sont utilisées.

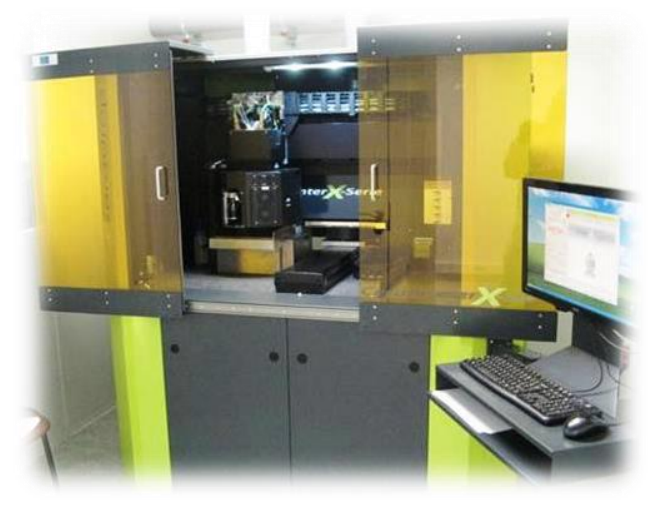

Figure 1 : CERADROP Xseries 2 têtes d'impressions avec la possibilité d'insoler par UV et de recuire par NIR. Environnement contrôlé en empoussièrement, température et hygrométrie. 
Première partie du TP ( 1 jour): Présentation de l'environnement de travail, des dispositifs à réaliser (jauges de contraintes organiques sur papier), description et prise en mains de l'équipement d'impression jet d'encre.

- Les étudiants " séjournent » en environnement contrôlé. En effet, certains paramètres ont une importance considérable sur la stabilité du processus d'impression.

- L'empoussièrement doit être limité afin d'assurer une surface d'impression la plus propre possible.

- L'hygrométrie doit être contrôlée afin d'éviter le bouchage des buses d'éjection dû a un séchage prématuré du solvant (composant principal d'une encre à base de nanoparticules d'argent par exemple).

- La température est un paramètre clef d'une bonne répétabilité de l'imprimabilité car sa variation entraine une variation de la viscosité des encres à éjecter.

- Les jauges de contraintes seront réalisées sur du papier et constituées d'électrodes à base de nanoparticules d'encre d'argent (Anapro 40LT15C) et recouvertes d'encre à base d'un polymère organique (PEDOT-PSS Clevios HC).

- Après une brève description de l'équipement (CERADROP X-series) une attention particulière est portée sur l'explication du mécanisme d'éjection associant technologies MEMS et microfluidique (figure 2).
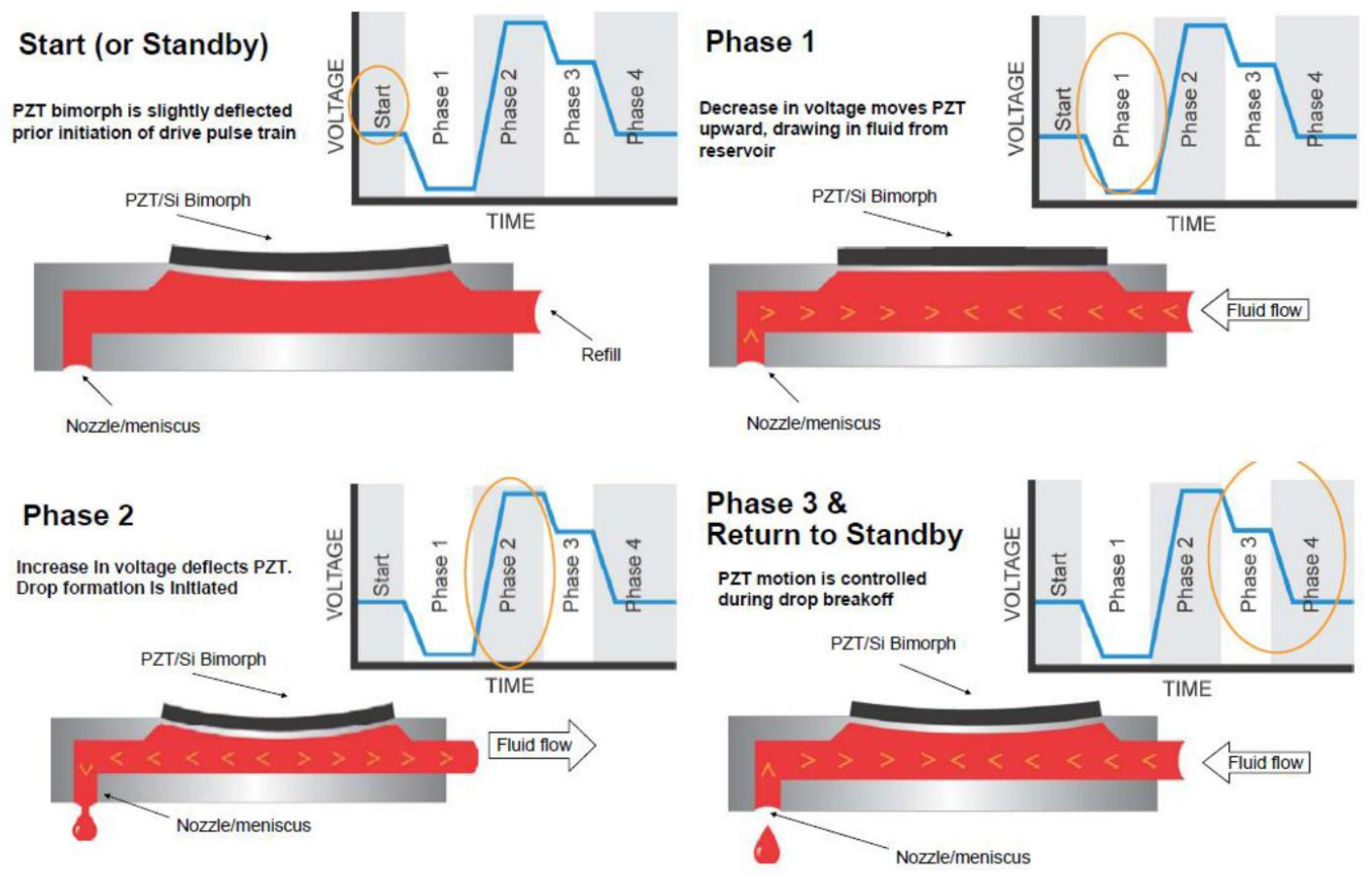

Figure 2 : Différentes étapes de fonctionnement du piézo [1]

- Etat initial : le respect d'un ménisque concave en sortie de buse est important pour assurer une bonne répétabilité. Si l'encre vient «mouiller » les parois externes de la buse l'éjection ne sera pas " correcte ». Le paramètre important est la tension de surface des liquides (optimum entre 28 et 33 $\mathrm{mN} / \mathrm{m})$.

- Phase $1: \Delta$ Pression $(\Delta \mathrm{P})$ négatif dans la chambre pour aspirer le liquide dans le réservoir suivi d'un temps de stabilisation. La tension appliquée sur le piézo ainsi que la durée de la phase doivent être 
ajustées jusqu'à ce que les deux ondes acoustiques créées aux extrémités se rencontrent au milieu de la chambre.

o Phase 2: Action sur le piézo pour initier la formation de la goutte. Le respect de la phase 1 permet d'éjecter une goutte avec le maximum d'énergie et par conséquent d'obtenir une vitesse maximum en sortie de buse. On peut aussi amener plus d'énergie en diminuant le temps de montée (pente plus raide) de cette phase.

○ Phase 3: $\triangle \mathrm{P}$ négatif dans la chambre pour créer la goutte en coupant " le doigt de liquide » en sortie de buse sous l'effet de la tension superficielle du liquide. La deuxième partie de cette phase permet « d'amortir le phénomène » et limite l'aspiration de bulle d'air dans la chambre.

○ Phase 4 : Etape de stabilisation avant le cycle suivant.

Deuxième partie du TP ( 2 jours) : Les deuxième et troisième jours du TP permettent aux étudiants de comprendre la démarche à adopter pour définir les paramètres d'impression. Cette démarche s'articulera suivant le synoptique ci-dessous (figure 3 ).

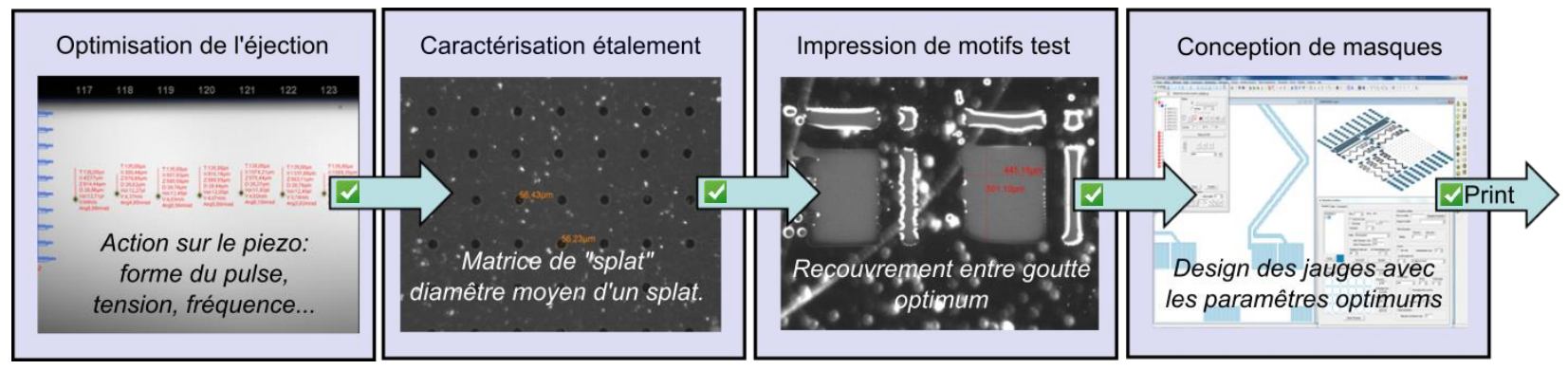

Figure 3 : Démarche permettant la qualification d'une encre, d'un substrat et l'optimisation de la conception des motifs désirés.

\section{Optimisation de l'éjection:}

Avant de pouvoir imprimer un motif il apparait évident qu'il faut ajuster les paramètres d'activation de l'élément piézoélectrique permettant de générer « une goutte bien formée ». Pour ce faire, l'équipement est muni d'un système de visualisation de l'éjection. Typiquement la vitesse d'une goutte éjectée est de $3 \mathrm{~m} \cdot \mathrm{s}^{-1}$ et peut atteindre $7 \mathrm{~m} \cdot \mathrm{s}^{-1}$. L'équipement $\mathrm{n}^{\prime}$ étant pas pourvu de caméra à images rapides pour des raisons de coût et d'encombrement, la visualisation de l'éjection d'une encre se fait par l'intermédiaire d'un système de visualisation (caméra et LED blanche) stroboscopique. Ainsi l'envoi de multiples flashs permet de décomposer un mouvement en une succession d'images superposées sur la même image donnant une impression de mouvement fluide!

Beaucoup de paramètres peuvent être ajustés pour optimiser l'éjection. La forme "d'onde " appliquée au piézo (figure 4) dans le cadre du TP comporte cinq paramètres importants, à savoir : le temps de monté $(\mathrm{tm})$, le temps de plateau (tp), le temps de descente (td), la fréquence d'éjection ( $f$ ) et la tension d'actuation (v). Ce signal relativement simple est suffisant car l'encre d'argent utilisée s'éjecte relativement facilement mais la "forme d'onde " peut être relativement plus compliquée (cf première partie du TP). L'optimisation de l'éjection n'étant pas l'objectif principal de ce TP, seule la fréquence et la tension d'actuation sont étudiées. 


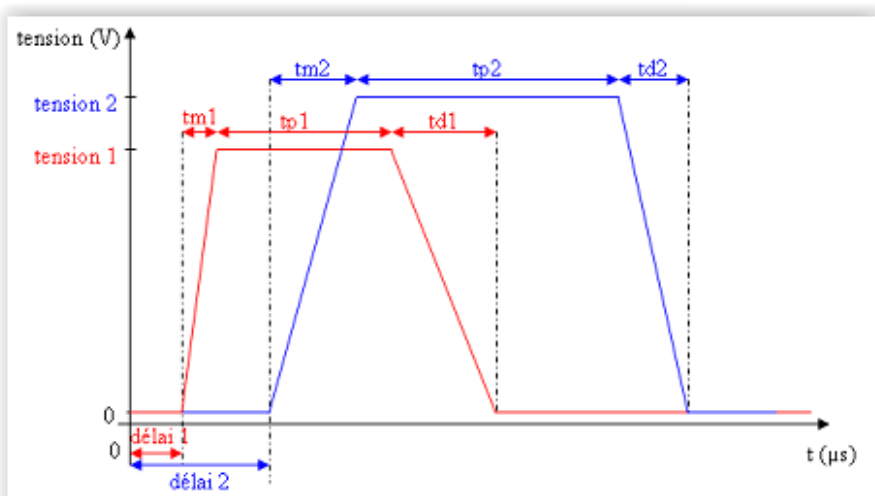

Figure 4 : Signal appliqué au piézo ; rouge pour les buses paires et bleu pour les buses impaires

\section{Action sur les paramètres}

- tension d'actuation (v) : La figure 5 montre que l'augmentation de la tension d'actuation provoque l'apparition de gouttelettes satellites pouvant altérer la définition du motif à imprimer.

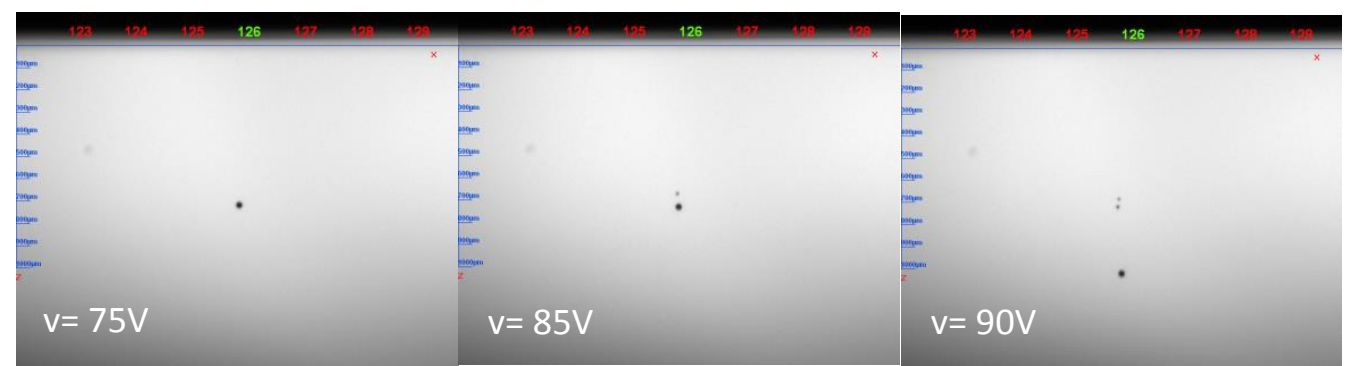

Figure 5 : Evolution de la qualité de l'éjection en fonction de la tension d'actuation du piézo

Les étudiants ont aussi accès à la mesure du volume et de la vitesse de la goutte et peuvent observer leurs dépendances vis-à-vis de la tension d'actuation du piézo. Dans le cas présent, une tension d'actuation de $75 \mathrm{~V}$ semble être adaptée à notre encre,

- fréquence de création des gouttes (f) : La fréquence d'éjection des gouttes est un paramètre clef qui permet d'évaluer la vitesse d'impression maximale. La figure 6 montre que la trajectoire du train de goutte généré par le piézo est dépendante de la fréquence d'éjection. 


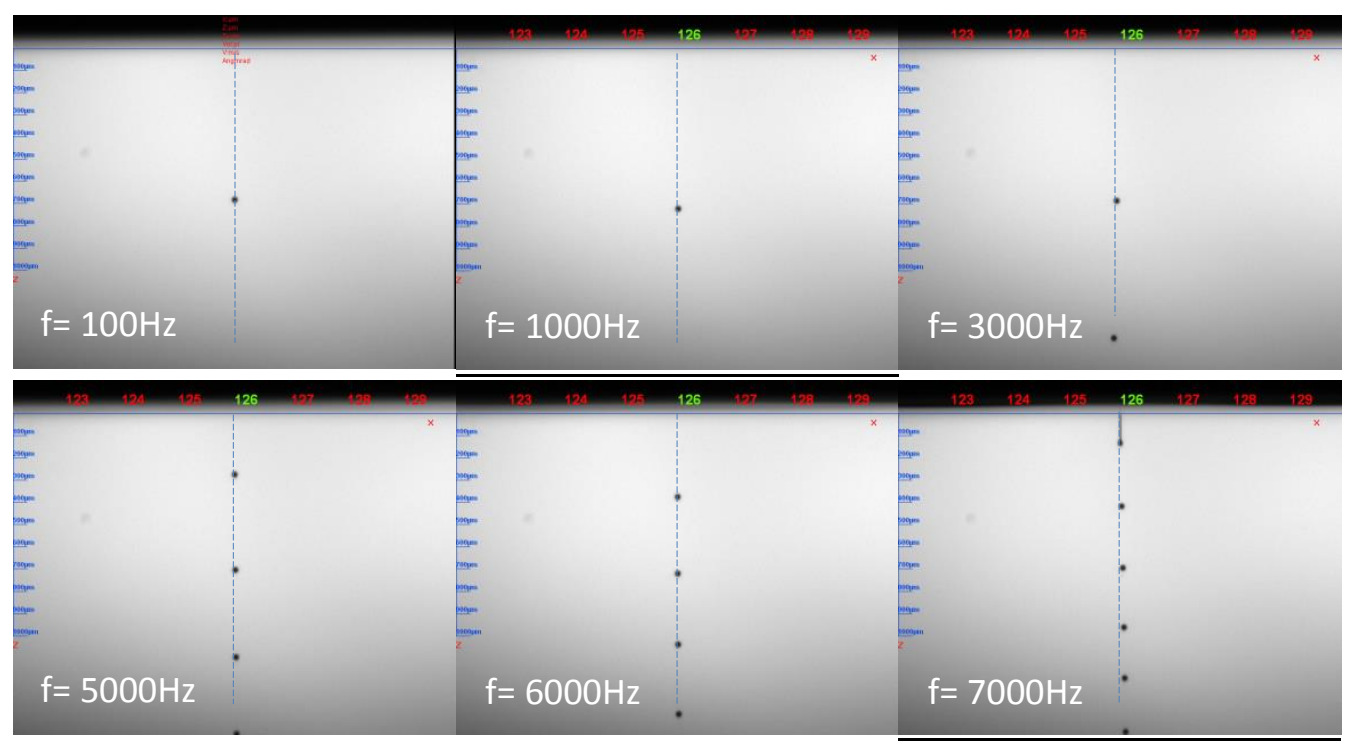

Figure 6 : Evolution de la qualité de l'éjection en fonction de la fréquence d'éjection

Dans le cas présent, une fréquence d'éjection maximale comprise entre 6 et $7 \mathrm{KHz}$ avec une tension d'actuation de $75 \mathrm{~V}$ semble être adaptée à notre encre.

\section{Caractérisation de l'étalement}

Après définition des bons paramètres d'éjections, les étudiants effectuent leurs premiers essais d'impressions sur le substrat papier.

Explication du mécanisme d'impression de motifs continus :

Un motif est composé d'une juxtaposition de gouttes (de volume avoisinant les 10pl) venant se déposer sur le substrat. En ajustant le pas entre gouttes jusqu'à coalescence de ces dernières, il est possible de définir un motif continu à la géométrie désirée. L'étalement d'une goutte sur un substrat donné dépend de paramètres tels que : la tension de surface des encres et de l'énergie de surface du substrat (la notion d'interaction liquide solide n'est pas détaillée faute de temps mais fera l'objet d'un TP complémentaire). Il faut donc déterminer pour chaque couple encre/substrat, le diamètre d'une goutte afin de fixer la distance entre goutte (interpénétration) permettant de créer des motifs continus. Pour ce faire, les étudiants imprimeront un motif composé d'une matrice de goutte d'encre déposée par pas de $200 \mu m$ (figure 5).

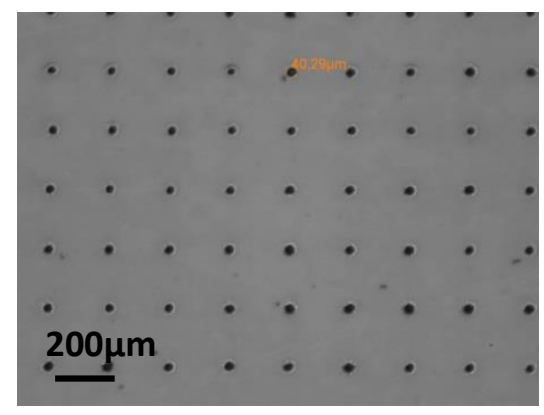

Figure 5 : Image du motif permettant de calibrer le diamètre d'une goutte unique déposée sur le substrat papier $(40 \mu \mathrm{m})$. La réalisation d'une matrice permet de rendre compte de la répétabilité de l'éjection. 
Impression de motifs test

L'impression de motifs tests permet d'ajuster l'interpénétration entre gouttes imprimées sur le substrat. Des motifs de calibration (figure 6(a)) sont donc imprimés à l'aide du logiciel CERASLICE développé par la société CERADROP et adaptés à la technologie d'impression jet d'encre. Les étudiants ont la possibilité de concevoir les motifs, paramétrer le remplissage des composants (mailler) en fonction des paramètres expérimentaux définis précédemment et de simuler le remplissage des motifs. Les étudiants ajustent donc l'interpénétration (figure 6(b)) grâce au logiciel et évaluent son impact après impression (figure 6(c)).
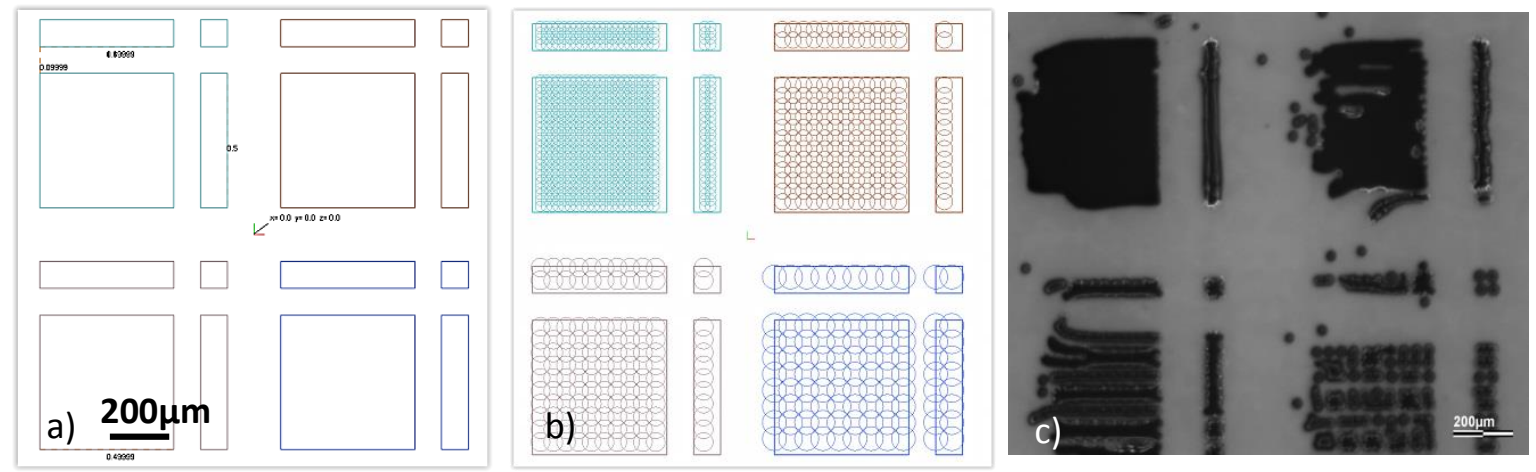

Figure 6 : (a) dimension des motifs de calibration; (b) simulation du remplissage d'un motif (chaque motif est maillé avec un paramètre d'interpénétration différent) ; (c) exemple d'impression

Dans le cadre du TP, le diamètre d'une goutte imprimée sur le substrat est de $40 \mu \mathrm{m}$.

Plusieurs cas de figure peuvent se produire :

- I'interpénétration est trop importante, le motif imprimé ne respecte pas les dimensions voulus car la quantité d'encre est trop importante,

- l'interpénétration est trop « faible ", le motif n'est pas continu,

- l'interpénétration est optimum, le motif respecte les dimensions.

\section{Conception des masques:}

La conception des masques s'effectuera à l'aide du logiciel CERASLICE. Les électrodes (figure 7) comportent : une zone de prise de contacts électriques, une distance inter-électrodes comprise entre 50 et $400 \mu \mathrm{m}$, des pistes aux formes complexes (courbes). Ce dernier point permet aux étudiants d'observer la différence de définition d'un motif " courbe " vis-à-vis d'un motif rectiligne réalisé dans le sens de passage de la tête d'impression.

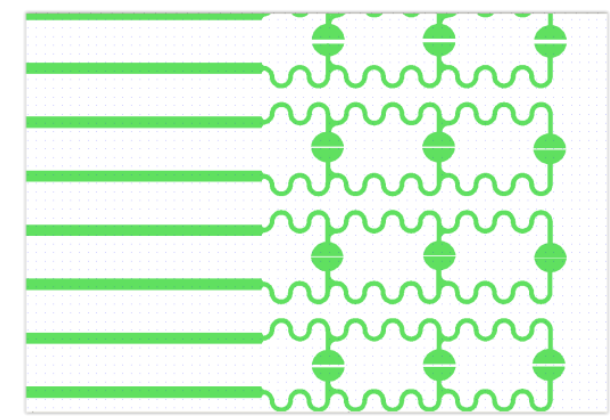

Figure 7 : Design du masque

$\underline{\text { Troisième partie du TP (1 jour) }}$ 
Réalisation des jauges de contraintes et caractérisation électrique sous déformation mécanique

\section{Impression des électrodes}

Les électrodes sont imprimées (figure 8(a)) et des mesures optiques permettent de rendre compte du respect du dimensionnel (figure $8(d)$ ). Les figures $8(b)$ et $(c)$ montrent le phénomène de crénelage apparaissant lors de la réalisation de formes non rectilignes.
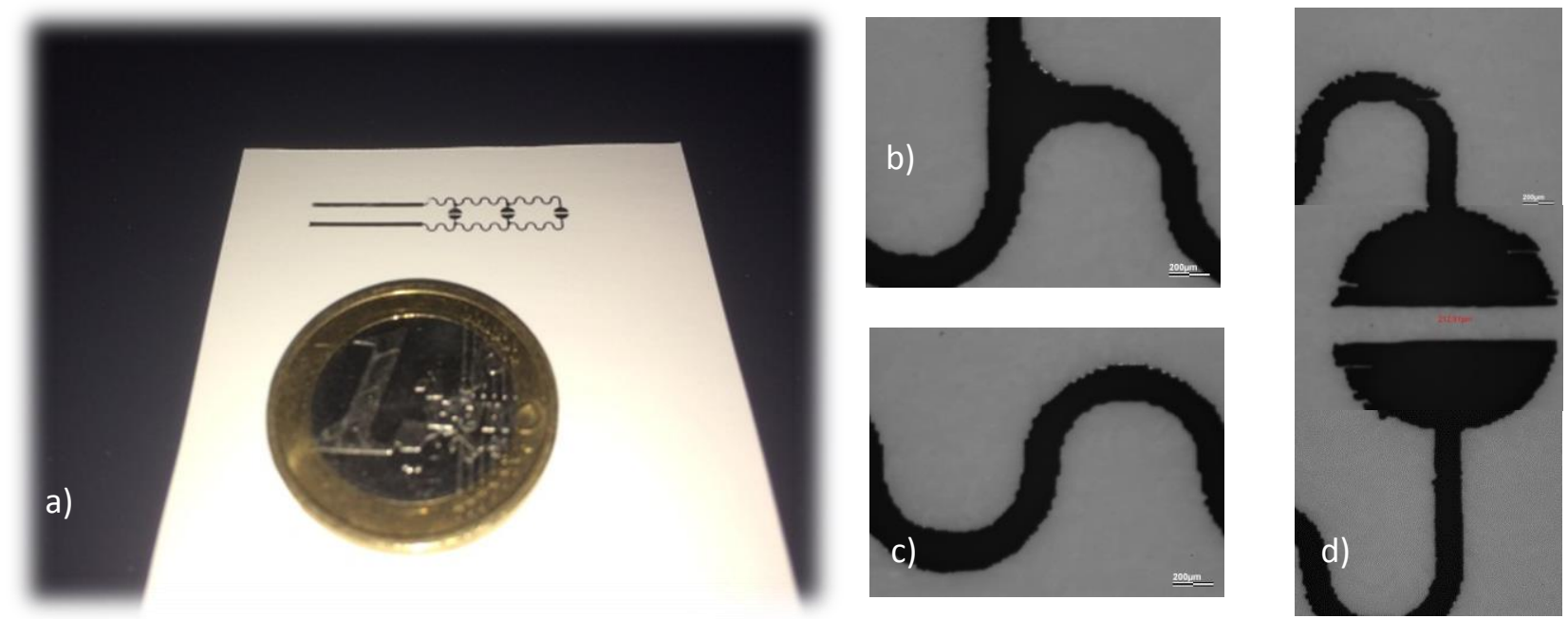

Figure 8 : (a) dimension des motifs de calibration; (b) simulation du remplissage d'un motif (chaque motif est maillé avec un paramètre d'interpénétration différent) ; (c) exemple d'impression

\section{Dépôts du PEDOT-PSS}

Le PEDOT-PSS sera déposé par la technique du spin-coating afin de sensibiliser les étudiants à une technique de dépôt très répandue dans l'industrie microélectronique.

\section{Caractérisation électrique sous contraintes mécaniques}

La caractérisation électrique (relevé courant-tension en figure 9) se fera par l'intermédiaire d'une station sous pointes. Des supports avec des rayons de courbures différents seront utilisés pour appliquer une déformation aux jauges de contrainte.
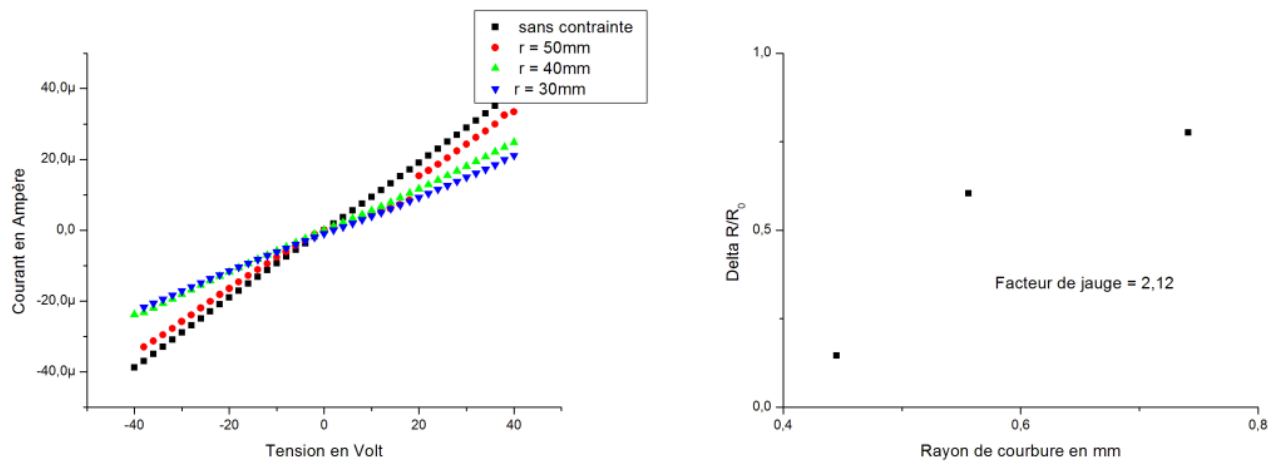

Figure 9: Exemple de relevés courant-tension d'une jauge de contrainte soumis à déformation et extraction du facteur de jauge.

\section{BILAN}


L'originalité de ce TP réside dans la technique de réalisation et la caractérisation d'un dispositif électronique simple sur substrat non conventionnel. L'impression par jet de matière est un sujet de recherche actuel et à le potentiel pour devenir une technologie de choix dans la réalisation d'étiquettes RFID, de circuits à base de transistors organiques pilotant des dispositifs d'affichages eux-mêmes organiques (OLED par exemple)... L'action mise en place par le CCMO permet donc aux étudiants de se familiariser avec cette thématique d'avenir.

[1] Scaling of Inkjet-Printed Transistors using Novel Printing Technique1s ; Huai-Yuan Tseng Technical Report No. UCB/EECS-2011-146 